\title{
Season of Birth Bias in Adult Pathology
}

\author{
Vladimir N Melnikov* \\ Research Institute of Physiology and Basic Medicine, Novosibirsk, Russia \\ *Corresponding author: Vladimir N Melnikov, Research Institute of Physiology and Basic Medicine, Novosibirsk, Russia
}

ARTICLE INFO
ABSTRACT

Received: 慧 February 03, 2020

Published: 㗀 February 11, 2020

Keywords: Month of Birth; Seasonality

Citation: Vladimir N Melnikov. Season of

Birth Bias in Adult Pathology. Biomed J Sci \&

Tech Res 25(3)-2020. BJSTR. MS.ID.004216.

\section{Opinion}

Perception of individual differences in vulnerability to pathogenic agents, as well as awareness of the need to predict such vulnerability, has a long history. However, it was not possible for a long time to determine a link between this vulnerability and exogenous and endogenous factors and to establish easily identifiable predictors of predisposition to certain disease and its outcomes. The cause of such situation was the lack, as we would say now, of long-term databases of medical and demographic data. However, this did not stop inquisitive researchers and ancient scientists and healers from summarizing and generalizing of scattered empirical observations and experience accumulated in an individual memory of physicians, archivists and recorded in ancient folios. The simplest and tempting individual sign then was and now is the month of birth (MOB), recorded in all documents accompanying a person through the life: birth records, medical records, biographies, and epitaphs.

A large body of scientific literature has been accumulated on the association between early developmental conditions and later life health outcomes. Epigenomic variation without affecting the underlying nucleotide sequences, occurring during the vulnerability time windows gametogenesis, placentation, fetal growth, and early postnatal period - is now known to serve a critical regulatory role in the developing and even adult organism. However, regardless of extending knowledge about the MOB-adult health association, this concept has some limitations and provides reasons for a skeptical attitude of some orthodox scientists to the link under discussion. This association is not a general phenomenon and is not similarly seen for every individual, potentially depending on the variable conditions influencing at early development: landscape, latitude, seasonal variations in climatic factors, the altitude above sea level, the mineral supply and the distance from marine coast, the presence of local geomagnetic anomalies transforming impacts of increased solar activity on humans, etc.

Since these influences on adult life and health deficits of elderly people are weak and later can be masked by other strong factors: narcosis and surgical operations, major infections, severe acute diseases, vaccinations, household and industrial poisons, harmful habits and environmental pollutions, substance use, vitamin and mineral deficiencies, x-rays medical inspection, etc. - this influence cannot be equally manifested in all persons. Moreover, even the same living conditions and risk factors, for example, in the household, exert different effects depending on the susceptibility and physiological responsiveness that are always different across individuals. This reason leads to the lack of the uniform relation and repeatability of the effects, which repeatability is usually considered an obligatory prerequisite for every truly scientific research. The clear principle impossibility of testing the relation in the controlled experiment might be one more objective reason for cautious attitude of the strong science to data on the fetal programming of adult life events. The fact is all scientific data about the analyzed relationship in human beings are obtained in retrospective descriptive studies with the observational design.

One more aspect to be discussed regards a possibility of transferring epidemiological data to individual level. How can 
practicing physicians use findings of population studies, having established the high probability of premature death from the certain disease, in their everyday activity at bedside? The fact of belonging of a given patient to the population at risk for a specific disease usually does not mean that this disease really exists or will being developed in the future. The use of probabilistic approach in medicine has been the topic of philosophical debates. It seems likely that epidemiologists and medical offices planning activities and making decisions in health care are the main customers of information on the MOB-association conception of interest.

With a slight shift of a few days, the division of the year into calendar months corresponds to 12-item differentiation by Zodiac signs. This gave rise to the astrological roots of mental, psychic, medical, and anthropological typologies, which turned out to be very close to horoscopes. This compromises the truly scientific approach to investigating the relationship of adult biomedical properties with the month and place of birth as a proxy for seasonal variation in physiological state or early environmental exposure at the stage of increased susceptibility, or "window of sensitivity", of the body to external influences.

The seeming simplicity of establishing associations tempts many uncritical scientists, who are inclined to mystical thinking and are not familiar enough with statistical methods of evidencebased medicine, to absolutize reliable and apparent results and expand their scope by introducing insufficiently valid facts into unpretentious journals and the mass consciousness. Additionally, it is very likely that some reports of association are false positive because comparing the MOB distribution seen in a group of cases with that expected based on averaged population or national birth statistics often turned out inadequate.

In light of the topic of interest, it is worth considering one more important item. A "fertile time" has passed for the achievements of medicine, when it quickly coped with major challenges as a result of technical progress and fundamental discoveries of biologists. Pathology caused by the action of strong factors: pathogens of major infections, cold, vitamin deficiency, food diatheses, physical stress, etc. - is currently well studied, ways for preventing and eliminating it are known and widely used in practice. The development of materials science, success in transplantation, surgery, immunology, and neonatology allowed practical medicine making significant progress in reducing health losses and increasing life expectancy. However, the potential of previous scientific discoveries in biomedicine regarding chronic non-communicable diseases was exhausted by the beginning of the XX1 century. Now one can state that the therapy of internal diseases lags behind diagnostics which is armed with modern means of visualization and localization of pathological processes, detection of biochemical anomalies and morphological evidences. Progress in the treatment of cancer, tuberculosis, neurodegenerative and mental diseases is still modest. Further success in increasing life expectancy through medical efforts will slow down and require more and more resources. This increases significance of weak causal and underlying pathological factors for any further progress in improving the effectiveness of primary prophylaxis and therapy. Among these weak factors are those of meteorological, geo- and heliophysic, electromagnetic, chronobiological nature. This certainly includes seasonally varying conditions of early development. The latter factor is especially important in northern regions with sharp continental climate and substantial differences between winter and summer conditions. Considering the significance of the season of birth as an efficient predictor which does not require large material resources to be implemented, one can consider this approach a useful tool for making a qualitative leap in coping with challenges facing modern healthcare.
ISSN: 2574-1241

DOI: 10.26717/BJSTR.2020.25.004216

Vladimir N Melnikov. Biomed J Sci \& Tech Res

(C) This work is licensed under Creative Commons Attribution 4.0 License

Submission Link: https://biomedres.us/submit-manuscript.php

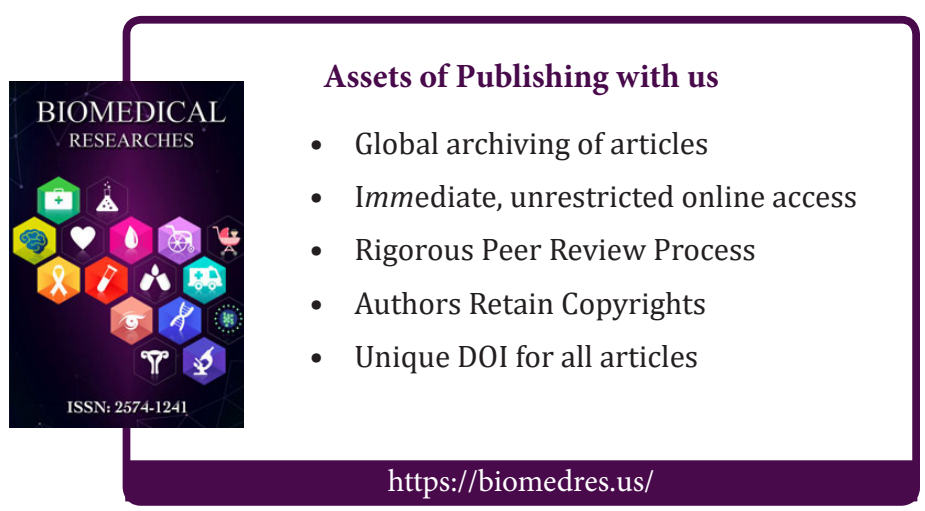

